\title{
Endometriosis Apendicular: una inusual localización de Endometriosis Extragenital
}

\author{
Felipe Arbeláez Casas,*; Angela María Ruiz Stemberg,**; Nidia Angélica Vásquez Trujillo****
}

\section{RESUMEN}

La endometriosis apendicular es un hallazgo infrecuente del compromiso extragenital de la enfermedad. Es usualmente asintomática, aunque puede generar un cuadro clínico de apendicitis. Su tratamiento, una vez realizado el diagnóstico, puede evitar complicaciones posteriores, ya sea por perforación o adherencias. La laparoscopia se constituye en una alternativa de elección para sumanejo, con una mínima morbilidad para la paciente.

PALABRAS CLAVES: Endometriosis, Apéndice, Laparoscopia

\section{ABSTRACT}

Apendicular endometriosis is an infrecuent finding of the extragenital compromise of the Llisesase. Usually asimptomatic, it can also produce apendicitis. Its treatment can avoid posterior complications as perforation or adhesions. Laparoscopy is a good alternative of treatment with minimal morbidity for the patient.

KEY WORDS: Endometriosis, Appendix, Laparoscopy.

\section{Introducción}

Dentro de la endometriosis extragenital, la del tracto gastrointestinal es la más frecuente. Según diversos autores se presenta entre el 25 al $40 \%$ de la pacientes con endometriosis pélvica y se asocia principalmente a los estados III y IV de la enfermedad $(1,2)$.

Teniendo en cuenta la teoria de la menstruación retrograda, implicada en la patogénesis de la endometriosis y considerando la proximidad del tracto digestivo con los órganos pélvicos, principalmente con las trompas de Falopio, es de esperar una asociación estrecha entre la endometriosis de los genitales internos y el compromiso gastrointestinal $(2,3)$.

En relạción con este principio, también podría suponerse que la endometriosis apendicular sea frecuente, pero sorprendentemente esto no ocurre y el hallazgo en esta localización es inusual $(4,5)$.

Sampson en 1921 fue el primero en describir la endometriosis apendicular en cuatro pacientes de una serie de doce con compromiso del tracto digestivo.

Posteriormente, series más grandes como la de Masson reportó que el $3 \%$ de la endometriosis gastrointestinal se

\footnotetext{
* Ginecólogo y Obstetra, Coordinador de la Unidad de Endoscopia Ginecológica.

* Ginecóloga y obstetra. Miembro de la Unidad de Endoscopia Ginecológica.

*** Residente III año Ginecología y Obstetricia.
}

ubicaba en el apéndice. Scott y Te Linde mostraron estudios donde la incidencia no superaba el 1,4\%, mientras Weedy y Ray en 1987 describieron una incidencia de hasta el $20 \%$ (6).

Estudios más recientes como los de Prystowsky, señalan en 85 pacientes con endometriosis gastrointestinal, una incidencia apendicular del $17 \%$ (4).

En nuestro medio no hay datos que puedan compararse, pues hasta el momento no se han realizado estudios que evalúen la incidencia de endometriosis apendicular.

En La unidad de Endoscopia Ginecológica del Hospital Infantil Universitario "Lorencita Villegas de santos" realizamos entre Noviembre de 1995 y Enero de 1999, 812 Laparoscopias. De estas, 201 que corresponde al 24.7\%, han mostrado endometriosis abdominopélvica. Solamente 4 , que es el $1,99 \%$, han tenido endometriosis apendicular, las cuales describimos a continuación:

caso 1

Paciente de 22 años, G0 P0, con cuadro progresivo de dismenorrea, dispareunia profunda y disquezia catamenial ocasional, de 2 años de evolución. En la evaluación física se encontró dolor a la palpación en fosa ilíaca izquierda. Los análisis paraclínicos, incluyendo ecografía pélvica transvaginal, fueron negativos.

Se sometió a laparoscopia encontrándose focos de endometriosis superficial ovárica, peritoneal y en la serosa 
apendicular. Se clasificó como endometriosis estado II (7). Se manejó con coagulación bipolar de focos pélvicos y posteriormente se prescribió $30 \mathrm{mg}$ diarios de acetato de medroxiprogesterona durante 6 meses. Se observó discreta mejoría del dolor según la clasificación de Biberoglu y Bergman (8). En la segunda mirada persistía la endometriosis grado II y se observó estenosis del tercio distal del apéndice por el foco de endometriosis de la serosa. Se realizó manejo de la enfermedad residual y apendicectomía laparoscópica, en un tiempo operatorio de 90 minutos, sin complicaciones. La paciente permaneció 48 horas hospitalizada. La anatomia patológica mostró focos de endometriosis en la subserosa apendicular con estenosis del lumen (figura 1.).

\section{Caso 2}

Paciente de 39 años, G1P1, con dolor pélvico crónico acíclico en fosa ilíaca derecha y dispareunia de 1 año de evolución. En el examen ginecológico se encontró una masa anexial izquierda de $5 \mathrm{~cm}$. de diámetro, móvil, no dolorosa. La ecografía transvaginal reveló una masa quística, de contenido ecóico, de $6 \mathrm{~cm}$. de diámetro mayor. El Ca 125 fue de $35 \mathrm{mU} / \mathrm{ml}$. En la laparoscopia se observó un endometrioma ovárico izquierdo, además de endometriosis superficial en el peritoneo, ovario, trompa derecha y en la superficie apendicular. Se clasificó como endometriosis estado IV. Realizándose cistectomía izquierda, coagulación de focos y apendicectomía, con un tiempo quirúrgico de 120 minutos, sin complicaciones.

Fue dada de alta a las 48 horas y el reporte de patologia confirmó el compromiso seroso del apéndice cecal. Posteriormente, recibió análogos de la GnRH por 6 meses y en la segunda mirada laparoscópica el estado de la enfermedad fue grado II.

\section{Caso 3}

Paciente de 33 años, G3 P2 A1, con cuadro de dolor pélvico crónico cíclico incapacitante, dispareunia y disquezia ocasional, de 3 años de evolución. Clínica y egográficamente se sospechaba endometriomas bilaterales. El Ca 125 era de $74.4 \mathrm{mU} / \mathrm{ml}$. En laparoscopia se encontró endometriomas bilaterales, con obliteración de fondo de saco posterior y compromiso apendicular. Se clasificó como endometriosis estado IV y se realizó adhesiolisis, cistectomía bilateral de los endometriomas y apendicectomía, en un tiempo quirúrgico de 120 minutos y sin complicaciones. En el estudio de anatomía patológica se confirmó la localización de la endometriosis a nivel del mesoapéndice. Se dió de alta a las 72 horas. Recibió análogos de la GnRH por 6 meses y en vista de la recidiva de la severa sintomatología y de las masas anexiales bilaterales fue sometida a histerectomía abdominal total con anexectomía bilateral.

\section{Caso 4}

Paciente de 37 años, G0P0, con cuadro de dolor pélvico crónico acíclico, y dismenorrea leve de 4 años de evolución. Al examen ginecológico se encontró una masa anexial derecha la cual fue confirmada por ecografía transvaginal, de $3.7 \mathrm{~cm}$. de diámetro mayor. El Ca 125 fue de $73.5 \mathrm{mU} /$ $\mathrm{ml}$. En la laparoscopia se observó endometriomas bilaterales, obliteración del fondo de saco posterior y proceso adherencial que involucraba el apéndice cecal, clasificándose como endometriosis estado IV. Se realizó cistectomía bilateral, adhesiolisis y apendicectomía en un tiempo quirúrgico de 110 minutos, sin complicaciones.

Permaneció hospitalizada por 48 horas. La anatomía patológica reveló focos de endometriosis en la serosa apendicular. La paciente se sometió a manejo con análogos de la GnRH durante 6 meses con mejoria de la sintomatología dolorosa y en la segunda mirada laparoscópica se clasificó la enfermedad como estado II.

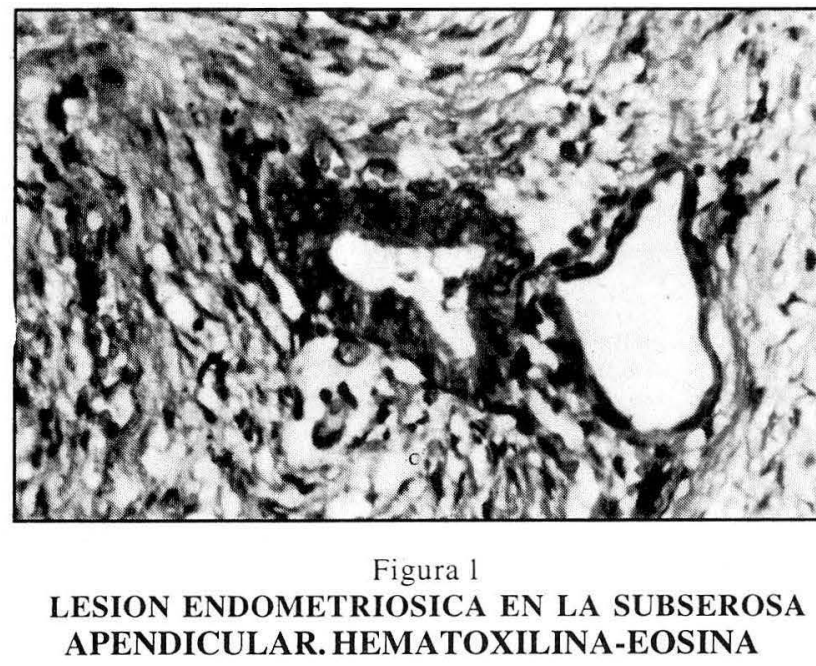

\section{Discusión}

La presentación clínica de la endometriosis apendicular es muy variada. La mayoría de la veces es asintomática y su diagnóstico se hace con base en los hallazgos intraoperatorios. Sus sintomas pueden estar enmascarados por la endometriosis pélvica concomitante (5).

En ocasiones y dependiéndo del grado de compromiso apendicular puede dar cuadros de apendicitis aguda, que si no son manejados a tiempo pueden terminar en procesos severos dados por ruptura y formación de absceso (5).

De la misma manera, puede manifestarse por cuadros crónicos con dolor en el cuadrante inferior derecho y sintomas digestivos de aparición catamenial. La intususcepción apendicular es una eventualidad clínica hasta en el $8 \%$ de los casos (9).

Otra forma inusual de presentación es la hemorragia masiva de un foco luminal apendicular, lo cual fue traducido en un cuadro de sangrado digestivo bajo en una mujer con dolor abdominal agudo y síntomas de compromiso hemodinámico (10).

Es notorio como en los casos reseñados por nosotros, 3 pacientes se presentaron con sintomas sugestivos de compromiso gastrointestinal. 
En cuanto a localización macroscópica, Mittal reporta el cuerpo apendicular como el sitio más frecuentemente comprometido ( $56 \%$ ) y su extremo distal en un $44 \%$.

Respecto a la localización histológica, el principal compromiso es sobre la muscular y seromuscular y en menor proporción sobre la serosa $(10,11)$, Sutton y Hardy atribuyen la sintomatología a la localización, tanto macroscópica como microscópica.

Nosotros hallamos que en el $75 \%$ de nuestras pacientes el compromiso fue de la serosa apendicular.

En cuanto al tratamiento hay consenso en que sí hay evidencia macroscópica de endometriosis apendicular, esta debe tratarse, con el fin de disminuir la tasa de complicaciones, tales como apendicitis aguda y procesos adherenciales severos (11).

La laparoscopia se ha convertido en el principal procedimiento diagnóstico y terapéutico de la endometriosis y como tal puede proveer un tratamiento adecuado para la endometriosis apendicular.

Semm en 1983 fue el primero en realizar una apendicectomía endoscópica completa. Mas adelante Leuken, con pocas modificaciones a la técnica anterior, publicó una serie de 47 casos en donde demostró tasas de morbilidad similar a la vía abierta con las innegables ventajas en relación con el tiempo quirúrgico, dolor postoperatorio, estancia hospitalaria, incapacidad y aspecto cosmético (12).

Posteriores autores han modificado la técnica quirúrgica acogiendo endoclips y suturas las cuales han modificado notoriamente los tiempos operatorios.

La técnica usada por nosotros incluye 2 ó 3 puertos accesorios de acuerdo a la condición de cada paciente, posterior disección del mesoapéndice con bipolar y sección hasta la base apendicular. Se realiza hemostasia cuidadosa de la arteria apendicular y se procede a la colocación de 3 endosuturas con material de resorción tardía, dejando $0,5 \mathrm{~cm}$. de espacio entre el 2 y el 3 endonudos para la coagulación y sección del especimen quirúrgico. El cabo del 3 nudo (el distal) se deja suficientemente largo para que sirva para la extracción, la cual se hace por el trocar de $10 \mathrm{~mm}$. Finalmente se realiza coagulación bipolar del muñón apendicular.

\section{Agradecimientos}

Muy especialmente al Doctor Ricardo Alvarado Pantoja quien con su entusiasmo, experiencia y dedicación sigue siendo ejemplo a las nuevas generaciones.

\section{Conclusiones}

El compromiso apendicular es usualmente un hallazgo casual en pacientes con endometriosis pélvica. Cursa asintomática, pero puede ocasionar cuadros agudos que originan morbilidad considerable. Esto debe ser muy tenido en cuenta ya que sus complicaciones se podrían sumar al efecto deletéreo que produce la endometriosis pélvica sobre la fertilidad de la paciente.

La laparoscopia constituye una gran herramienta diagnóstica y terapéutica en la endometriosis pélvica y también puede serlo en el manejo de la endometriosis apendicular, ya que posee las características de ser un procedimiento efectivo y seguro en manos experimentadas, sin originar mayor morbilidad.

\section{BIBLIOGRAFIA}

1. Jubanyik K J, Comité F. Exh.apelvic Endometriosis. Obsmt Gynecol Clin North Am, 1997; 24:411-440. .

2. Markham S M, Carpenter S E, Rock J A. Extrapelvic Endometriosis. Obstet Gynecol Clin North Am, 1997;24:260-275.

3. Oral E, Arici A. Pathogenesis of Endometriosis. Obstet Gynecol Clin North Am. 1997;24:219-231.

4. Prystowsky J B, Strykers J, Ujiki G T et al. Gastrointestinal Endometriosis. Arch Surg. 1988;123:855-857.

5. Mittal N K, Choudhury S P, Cortez J A. Endometriosis of the Appendix Presenting as acute Appendicitis. Am J Surg, 1981;142:519-521.

6. Weew J C, Ray J E. Endometriosis of the Bowel. Obstet Gynecol. 1987; 69:727-730.

7. The American Fertility Society. Revised Classification of endometriosis:1985. Fertil Steril 1985;43:351-354.
8. Biberoglu P, Berghman J, Pain Classification of Endometriosis. Am J Obstet Gynecol, 1981; 139: 645-654.

9. Panzer S, Pitt H A, Wallach E E, Intussuuception of the appendix due to endometriosis. Am J Gastroenterol. 1995;90:1892-1893.

10. Shome G P, Nagaraju M,Munis A. Appendiceal Endometriosispresenting as massive Lower intestinal hemorrhaeg. Am J Gastroenterol.1995:90:1881-1883.

11. Pitáaway D, Appendectomy in the Surgical Treatment of Endometriosis. Obstet Gy necol, 1983;61:421-423.

12. Lueken R P, Moeller C P, Heeckt B. Review of 47 Laparoscopic Appendectomies. J Am Assoc Gynecol Laparosc. 